\title{
Cytarabine, Venous Catheter Removal, Sepsis, Diagnosis of Malignancy, and Takotsubo Syndrome
}

\author{
John E. Madiasa \\ Reply: Stefan Baumann ${ }^{b}$ \\ a Icahn School of Medicine at Mount Sinai, New York, NY, and the Division of Cardiology, Elmhurst Hospital Center, Elmhurst, NY, USA; \\ ${ }^{b}$ First Department of Medicine, University Medical Center Mannheim, University of Heidelberg, Mannheim, Germany
}

\section{To the Editor}

The report by Baumann et al. [1], published in the September, 2014 issue of Oncology Research and Treatment, about the 58-year-old man with acute myeloid leukemia who suffered Takotsubo syndrome (TTS) while receiving the second course of consolidation chemotherapy with intravenous cytarabine (CYT), underscores the complexity of exploring plausible trigger(s) of TTS in patients with malignancies. Instead of delving into the issue of a possible 'specific' cardiotoxic effect of CYT, one should rather consider the 'nonspecific' emotional impact of having been diagnosed with a malignancy. Malignancies are common in patients with TTS, and consequently have prompted some to advocate that patients developing TTS should be evaluated for the presence of underlying malignancy [2-5]. Certainly, sepsis, procedures (e.g., removal of a central venous catheter), and other physical stresses should be also considered as triggers, but one wonders about their direct impact, in contrast to the indirect inciting effects on the autonomic sympathetic nervous system representation sites of the brain. Baumann and colleagues refer to the basal cardiac hypercontractility as 'compensatory'; however it is possible that this is 'part and parcel' of TTS, and not a response to the apical and midventricular hypokinesis/akinesis/dyskinesis. Also they are citing 'catecholamine-induced microvascular vasospasm', as a plausible cause of TTS, while direct catecholamine cardiomyocyte toxicity should be considered as well.

John E. Madias, MD, FACC, FAHA

\section{Reply by Baumann et al.}

Madias criticized in his letter to the editor that we underrate the difficulty of exploring possible triggers of Takotsubo cardiomyopathy (TC) in patients with leukemia in our recently published case report [1]. It is indisputable that causative factors for a TC are multifarious and therefore it is often difficult to identify a clear principle. We agree with Dr. Madias' comment that in our case several potential triggers were present, such as late effect of cardiotoxic agents, sepsis, or emotional stress, all of which might have been the origin for the highly reduced left ventricular function. The association of TC with malignancies is undisputed, whereas the underlying pathophysiologic mechanism remains unclear. The stress of receiving the life-threatening diagnosis of cancer or an aggravation of the cardiac adrenoceptor sensitivity might be the origin [3].

Dr. Madias is right by raising the objection that we did not focus on specific cardiotoxic side effects of a single chemotherapeutic. However, in addition to listing and discussing all possible triggers for TC in detail, an important aspect was to make this complex disease pattern more known among clinical oncologists, who might not be familiar with this uncommon disease and consider TC into their future differential diagnosis.

Stefan Baumann, M.D.

\section{Disclosure Statement}

The authors declare no conflicts of interest.

\section{References}

1 Baumann S, Huseynov A, Goranova D, Faust M, Behnes M, Nolte F, Heidenreich D, Hofmann WK, Borggrefe M, Akin I, Klein S. Takotsubo cardiomyopathy after systemic consolidation therapy with high-dose intravenous cytarabine in a patient with acute myeloid leukemia. Oncol Res Treat. 2014;37:487-90.
Angelini P, Holoye PY. Neurocardiogenic syncope andPrinzmetal'sangina associated with bronchogenic carcinoma. Chest 1997;111:819-22.

3 Burgdorf C, Kurowski V, Bonnemeier H, Schunkert H, Radke PW. Long-term prognosis of the transient left ventricular dysfunction syndrome (Tako-Tsubo cardiomyopathy): focus on malignancies. Eur J Heart Fail 2008;10:1015-9.
4 Burgdorf C, Nef HM, Haghi D, Kurowski V, Radke PW. Tako-tsubo (stress-induced) cardiomyopathy and cancer. Ann Intern Med 2010;152:830-1.

5 Angelini P. Neurocardiogenic syncope and cancer: a paraneoplastic association? Arch Intern Med 1999;159: 2484.

\section{KARGER \\ Fax +497614520714

\section{(c) 2015 S. Karger GmbH, Freiburg}

2296-5270/15/0383-0125\$39.50/0
John E. Madias, MD, FACC, FAHA

Division of Cardiology

Elmhurst Hospital Center

79-01 Broadway, Elmhurst, NY 11373, USA

madiasj@nychhc.org 